\title{
Vertigo: A single Institutional Experience
}

\author{
Aso Sabir Saeed $(\operatorname{FRCP}(\operatorname{Ed}))^{1}$
}

Abstract

Background: Vertigo is an illusion or hallucination of movement, often horizontal and rotatory, either of oneself or the environment. The type is either central or peripheral, but sometimes it cannot be identified.

Objective: We analyzed the etiologies of peripheral and central vertigo in our patients. Patients and Methods: The cross-sectional observational study enrolled 100 patients consecutively, who had attended the Rizgary Teaching Hospital's neurology outpatients' clinic in Erbil, Iraq, from January 1, 2019, to October 31, 2019. Thorough history, full general and neurological examinations and an extensive battery of investigations (including cranial contrast MRI) were performed. Patients with peripheral vestibulopathy and vertigo were assessed by otolaryngologists.

Results: Females $(69 \%)$ outnumbered males $(31 \%)$. Patients in their $6^{\text {th }}$ decade were the commonest target; the mean age was 46.7 years. Peripheral vertigo was evident in $46 \%$ of the patients while central vertigo occurred in $36 \%$ of them. In $18 \%$ of the patients, no cause was identified; most of them were females $(88.8 \%)$ in their $7^{\text {th }}$ decade of age. Benign paroxysmal positional vertigo (BPPV) dominated the peripheral vertigo cases. The most prevalent cause of central vertigo was vertebrobasilar insufficiency (VBI) (27\%); many were above 50 years of age.

Conclusion: Peripheral vertigo was more prevalent than the central ones. BPPV and VBI were the commonest causes of vertigo, in general. Vertigo mainly targeted middle-aged individuals in our city.

Keywords:Vertigo,Vestibulopathy, Vertebrobasilar insufficiency,Benign paroxysmal positional vertigo.

Corresponding Author: drasobzeni@yahoo.com

Received: $23^{\text {th }}$ December 2019

Accepted: $12^{\text {th }}$ March 2020

DOI:https://doi.org/10.26505/DJM.19015111223

\footnotetext{
${ }^{1}$ Kurdistan Council for Medical Specialties - Erbil- Iraq
}

\section{Introduction}

Vertigo is defined as an illusion or hallucination of movement, often horizontal and rotatory, either of oneself or the environment.Associated nausea and vomiting suggest a peripheral rather than central
cause[1-3].Symptoms of vertigo account for 10.7 visits per 1000 persons per years in general practice morbidity statistics [4]. A through history and physical examination usually differentiate between genuine vertigo 
from pseudo-vertigo in patients who have been experiencing dizziness or lightheadedness. This results from lesions of the visual, somatosensory, or vestibular systems. Visual vertigo is caused by new or incorrect spectacles or by the sudden onset of an extraocular muscle paresis with diplopia; in either instance, CNS compensation rapidly counteracts the vertigo. Somatosensory vertigo, rare in isolation, is usually due to a peripheral neuropathy or myelopathy that reduces the sensory input necessary for central compensation when there is dysfunction of the vestibular or visual systems. The most common cause of pathologic vertigo is vestibular dysfunction involving its end organ (labyrinth), nerve, or central connections[4,5].

\section{Patients and Methods}

This cross-sectional observational study enrolled 100 patients consecutively, who had attended the Rizgary Teaching Hospital's neurology outpatients' clinic in Erbil, Iraq, from January 1, 2019, to October 31, 2019. Both genders were included, and their ages ranged from 11 to 77 years. Thorough history was taken and only patients with true vertigo were included, and full general and neurological examinations (including rapid head impulse test and DixHallpike maneuvers) were performed by neurologists and neurology trainees. Rapid head impulse test and Dix-Hallpike maneuvers were conduced by neurologists and neurology trainees, according to their standards[5].
Complete blood counts, erythrocyte sedimentation rate, blood urea and serum electrolytes, blood glucose, serum lipids, liver function tests, and TSH were obtained in all patients. Electroencephalography (EEG) and cranial magnetic resonance imaging (MRI) were done in patients who have been suspected of having vertiginous migraine or seizures. Radiological tests like X-ray of mastoids, cervical spines, CT scan and MRI of head were done when required, according to the clinical presentation and findings. Brain CT scan and/or MRI were also ordered when a central cause was suspected (2 patients had cranial CT scan and 72 patients had cranial MRI). The rest of patients $(n=26)$ were not examined by cranial CT scan or MRI, as the causes of vertigo were obvious.

Patients with vertigo of peripheral origin were selected and sent to otolaryngologists for further evaluation. Follow-up consisted of direct telephone contact with each patient to evaluate response to treatment and set appointments for regular visits. Contact also occurred on a regular basis over the study period in order to re-evaluate the response after completing the investigation or after having performed the otolaryngologists' consultation.

\section{Statistical analysis}

Data were analyzed by an independent statistician using the statistical package for social sciences (SPSS), version 21.0.

\section{Results}

There was a female preponderance with a female to male ratio of 2.2: 1 (there were 69 
females and 31 males). Their ages ranged from 11 to 77 years. The mean age was 46.7 years and the peak occurrence of vertigo was in the $6^{\text {th }}$ decade Table (1).

Table (1): Gender and age distribution $(\mathrm{n}=100)$

\begin{tabular}{||c||c||c|c||}
\hline Age (year) & Male & Female & $\begin{array}{c}\text { Total number and } \\
\text { percentage (\%) }\end{array}$ \\
\hline \hline $0-10$ & 0 & 0 & 0 \\
\hline \hline $11 \_20$ & 5 & 1 & 6 \\
\hline \hline $21-30$ & 3 & 12 & 15 \\
\hline \hline $31-40$ & 8 & 12 & 20 \\
\hline \hline $41-50$ & 5 & 8 & 13 \\
\hline \hline $51-60$ & 4 & 18 & 22 \\
\hline $60-70$ & 4 & 15 & 19 \\
\hline \hline $70-77$ & 2 & 3 & 100 \\
\hline \hline TOTAL & 31 & 69 & \\
\hline
\end{tabular}

Table (2) demonstrates the types of vertigo; central one, with mean ages of 52.7 years and central, peripheral, and unidentified. The 39.5 years respectively.

Incidence of peripheral vertigo was more than

Table (2): Types of vertigo in both genders $(n=100)$

\begin{tabular}{||l||c||c||c||}
\hline \hline \multicolumn{1}{|c||}{ VERTIGO TYPE } & Males & & \\
\hline \hline Peripheral & 18 & Females & TOTAL \\
\hline \hline Central & 12 & 24 & 46 \\
\hline \hline Unidentified & 2 & 16 & 36 \\
\hline \hline
\end{tabular}

Table (3) shows the causes of vertigo. The commonest cause was VBI $(n=27)$ while the vestibular schwannoma (one patient for least etiology was multiple sclerosis and

Table (3): Causes of vertigo $(n=100)$

\begin{tabular}{||l|c||}
\hline \multicolumn{1}{|c|}{ Causes } & Number of patients \\
\hline \hline VBI & 27 \\
\hline \hline BPPV & 25 \\
\hline \hline Vestibular Neuritis & 18 \\
\hline \hline Vertiginous Migraine & 15 \\
\hline \hline Meniere's disease & 5 \\
\hline Epilepsy & 5 \\
\hline Multiple sclerosis & 3 \\
\hline \hline Vestibular Schwannoma & 1 \\
\hline \hline TOTAL & 100 \\
\hline * VBI, vertebrobasilar insufficiency; BPPV, benign paroxysmal positional vertigo
\end{tabular}


Table (4) shows gender distribution of vertigo due to VBI. Most patients were in their $5^{\text {th }}$ and $6^{\text {th }}$ decades of life. More than
$85 \%$ of those patients were above the age of 50 years.

Table (4): Age distribution of vertigo due to vertebrobasilar insufficiency $(n=27)$

\begin{tabular}{|c|c|}
\hline Age (year) & Number of patients \\
\hline $0-10$ & 0 \\
\hline $11 \_20$ & 0 \\
\hline $21-30$ & 1 \\
\hline \hline $31-40$ & 2 \\
\hline $41-50$ & 1 \\
\hline \hline $51-60$ & 10 \\
\hline \hline $60-70$ & 9 \\
\hline \hline $70-77$ & 4 \\
\hline \hline TOTAL & 27 \\
\hline
\end{tabular}

Table (5) shows vertigo due to unidentified The peak incidence was in the $7^{\text {th }}$ decade of causes, which consisted of 18 patients (18\%). life.

Table (5): Unidentified causes $(n=18)$

\section{Discussion}

\begin{tabular}{|c|c|}
\hline Age (year) & Number of patients \\
\hline \hline $0-10$ & 0 \\
\hline $11 \_20$ & 1 \\
\hline $21-30$ & 3 \\
\hline $31-40$ & 0 \\
\hline $41-50$ & 1 \\
\hline $51-60$ & 4 \\
\hline \hline $61-70$ & 8 \\
\hline \hline $71-77$ & 1 \\
\hline \hline TOTAL & 18 \\
\hline \hline
\end{tabular}

Several mechanisms are responsible for the maintenance of a balanced posture and for awareness of the position of the body in relation to its surroundings. The most important of the afferent impulses (which inform us of the position of different parts of the body, in response to these impulses, the adaptive movements necessary to maintain equilibrium are carried out) are the
1. Visual impulses from the retinae and possibly proprioceptive ones from the ocular muscles, which enable us to judge the distance of objects from the body.

2. Impulses from the labyrinths, which function as highly specialized spatial proprioceptors and register changes in the velocity of motion (either acceleration or deceleration) and position of the body. following: 
3. Impulses from the proprioceptors of the joints and muscles are essential to all reflex, postural, and volitional movements.

The sense organs listed above relate to the cerebellum and with certain ganglionic centers and pathways in the brainstem, particularly the vestibular nuclei, and, via the medial longitudinal fasciculi, with the ocular motor nuclei. Old people may lose their balance on extending the neck, and their peripheral sensory afferents are often impaired, as are the protective postural mechanisms, making falls more frequent[5].

Differentiating genuine vertigo from pseudo-vertigo is critical. On the other hand, differentiating episodes of physiological vertigo from pathological vertigo is important in managing and treating such patients. Genuine vertigo should be classified as central or peripheral vestibulopathy, as the diagnostic approach is quite different. In addition, differentiating vertigo from other causes of lightheadedness or dizziness should not be forgotten[5-12]. Some investigations can confidently diagnose the etiology of vertigo and its site[13,14].

Peripheral vertigo is the most common type of true vertigo [14]. BPPV is the most common cause of vertigo in adults $[15,16]$. In general, BPPV together with acute vestibular neuritis, and Ménière's disease cause most cases of vertigo; bedside test can help physician with this regard[17,18]. The use of cranial MRI in the diagnostic workup of vertigo is not a routine one. It depends on the symptomatology of the patient and his/her clinical findings[19].
Vertigo is a common symptom, which may be highly disabling. It should be kept in mind that vertigo ranks $2^{\text {nd }}$ on the list of reasons of visiting emergency rooms, neurologists, and otolaryngologists. Most patients can be diagnosed and managed on outpatients' basis, with a good clinical outcome[20].

In our 100 cases, the gender difference was visible; females $(69 \%)$ were more than males (31\%). This is consistent with a study by Min Yin et al in Japan [21] and a study by Marina Titlic et al in Croatia [22]. About $89 \%$ of the cases were between 21 to 70 years of age, and the peak incidence of vertigo was in the 6th decade of life; this is comparable to Marina Titlic et al study [22]. The mean of the age of patients was 46.7 years.

The disease was less common below 20 years and above 70 years of age. A study by Debasish Burman et al showed that about $92.6 \%$ of their cases were between 21-60 years of age, and that the disease was less common above the age of 50 years and was rare below 20 years of age[23].

We found that peripheral vertigo was more common than central one, and this is consistent with the Min Yin et al study [21]. In our study, no clear cause was found (the cause was unidentified) in $18 \%$ of cases; this result is also consistent with the Min Yin et al study [21]. Lee $\mathrm{H}$ and colleagues studied prospectively 72 cases of vertigo of unknown cause and attributed some of them to migraine; their results suggested that migraine should be considered in the differential diagnosis of isolated recurrent vertigo of unknown cause[24]. The mean age 
of patients with central vertigo was 52.7 years and was more than that of peripheral vertigo (39.5 years); this indicates that compared to younger patients, elderly patients have a high tendency of developing central vertigo [21]; this is probably because of the higher rate of atherosclerotic changes and more degenerative changes of cervical spine which is basis for VBI (25-27). This result is comparable with the Marina Titlic et al study [22].

The most common cause of vertigo in our study was VBI (27\%), comparable to the Marina Titlic et al study (36.5\%) [22], followed by BPPV. This is because our patients were collected in the only available neurology department in the whole governorate, where all neurological cases are referred to. Most patients were in their 5th and 6 th decades of life. More than $85 \%$ of those patients were above the age of 50 years, because older age is a recognized risk factor for atherosclerosis and VBI [6].

\section{Conclusions}

Peripheral vertigo was more prevalent than central ones. BPPV and VBI were the commonest causes of vertigo, in general. Vertigo mainly targeted middle-aged individuals, mostly females, in our city.

\section{Recommendations}

The presence of vertigo in middle-aged individuals should always prompt the physician search for a central cause, at first, which may be disabling or life-threatening. Peripheral vertigo is better to be assessed by otolaryngologists.

\section{References}

[1]Baloh R, Honubia V, Jacobson K. Benign positional vertigo: clinical and oculographic features in 240 cases. Neurology. 1987;37:371-8.

[2]Yardley L, Luxon L, Haacke N. A longitudinal study of symptoms, anxiety, and subjective wellbeing in patients with vertigo. Clin Otolaryngol. 1994;19:109-16.

[3] J Kanagalingam, D Hajioff, S Bennett. 10-minute consultation, Vertigo, BMJ. 2005;330: 523.

[4]Mc Cormick A, Fleming D, Charlton J. Morbidity Statistics from General Practice; Fourth National Study, 1991-1992. London, UK: Office of Population Censuses and Surveys; 1995.

[5]Ropper AH, Samuel MA, Klein JP, Prasad

S. Adams and Victor's Principles of Neurology, 11th Edition. New York, USA: McGraw-Hill Education; 2019.

[6]Jameson JL, Fauci AS, Kasper DL, Hauser SL, Longo DL, Loscalzo L. Harrison's Principles of Internal Medicine, 20th Edition. New York, USA: McGraw-Hill Education; 2019.

[7]Kroenke K, Lucas CA, Rosenberg ML, et al. Causes of persistent dizziness. A prospective study of 100 patients in ambulatory care. Ann Intern Med. 1992;117:898-904.

[8]Derebery MJ. The diagnosis and treatment of dizziness. Med Clin North Am. 1999;83:163-77.

[9]Brandt T, Bronstein AM. Cervical vertigo. J Neurol Neurosurg Psychiatry. 2001;71:812. 
[10]Tusa RJ, Herdman SJ. Diagnosis and treatment of the dizzy patient. Hospital Physician 1997;33:22-37.

[11]Solomon D. Distinguishing and treating causes of central vertigo. Otolaryngol Clin North Am. 2000;33:579-601.

[12]Furman JM, Marcus DA, Balaban CD. Migrainous vertigo: development of a pathogenetic model and structured diagnostic interview. Curr Opin Neurol. 2003;16:5-13. [13]International Headache Society. The international classification of headache disorders. Cephalalgia. 2018;38 (3rd edn.):1211.

[14]Bhidayasiri R, Waters MF, Giza CC. Neurological Differential Diagnosis. A prioritized Approach. London, UK: WileyBlackwell; 2005.

[15]Honrubia V, Baloh RW, Harris MR, Jacobson KM. Paroxysmal positional vertigo syndrome. Am J Otol. 1999;20:465-70.

[16]Baloh RW, Jacobson K, Honrubia V. Horizontal semicircular canal variant of benign positional vertigo. Neurology. 1993;43:2542-9.

[17]Labuguen RH. Initial evaluation of vertigo. Am Fam Physician. 2006;73(2):24451.

[18]Halmagyi GM, Curthoys IS. A clinical sign of canal paresis. Arch Neurol. 1988;5:737-9.

[19]Baloh RW. Differentiating between peripheral and central causes of vertigo. Otolaryngol Head Neck Surg. 1998;119(1):55-9.
[20]Brandt T, Dieterich M, Strupp M. Vertigo and Dizziness. New York, USA: Springer; 2005.

[21]Yin M, Ishikawa K, Wong H, Shaibata Y. A clinical epidemiological study in 2169 patients with vertigo. Auris Nasus Larynx. 2009;36(1):30-5.

[22]Titlic M, Tonkic A, Jukic I, Capkun V, Aleksic-Shihabi A. Vertigo in Neurological Practice. Acta Clin Croat. 2007;46:305-9. [23]Burman D, Goswami S, Majumdar PK. A study on peripheral vertigo in a Kolkata based hospital. Indian J Otolaryngol Head Neck Surg. 2002;54(2):101-4.

[24]Lee H, Sohn SI, Jung DK, et al. Migraine and isolated recurrent vertigo of unknown cause . Neurol Res. 2002;24(7):663-5.

[25]Wolf PA, Abbot RD, Kannel WB. Atrial fibrillation as an independent risk factor for stroke: the Framingham study. Stroke. 1991;22(8):983-8.

[26]Reiner Z, Tedeschi-Reiner E. New information on pathophysiology of atherosclerosis. Lijec Vjesn. 2001; 123:2621.

[27]Kerber KA, Brown DL, Lisabeth LD, Smith MA, Morgenstern LB. Stroke among patients with dizziness, vertigo, and imbalance in the emergency department: a population-based study. Stroke. 2006;37(10):2484-7. 